\title{
Time-dependent density functional theory for the charging kinetics of electric double layer containing room-temperature ionic liquids
}

Cheng Lian, Shuangliang Zhao, Honglai Liu, and Jianzhong Wu

Citation: The Journal of Chemical Physics 145, 204707 (2016);

View online: https://doi.org/10.1063/1.4968037

View Table of Contents: http://aip.scitation.org/toc/jcp/145/20

Published by the American Institute of Physics

\section{Articles you may be interested in}

Relationship between pore size and reversible and irreversible immobilization of ionic liquid electrolytes in porous carbon under applied electric potential

Applied Physics Letters 109, 143111 (2016); 10.1063/1.4964130

Ion distribution and selectivity of ionic liquids in microporous electrodes

The Journal of Chemical Physics 146, 174701 (2017); 10.1063/1.4982351

Evaluation of the constant potential method in simulating electric double-layer capacitors

The Journal of Chemical Physics 141, 184102 (2014); 10.1063/1.4899176

Structures of hard-sphere fluids from a modified fundamental-measure theory

The Journal of Chemical Physics 117, 10156 (2002); 10.1063/1.1520530

Evaluation of molecular dynamics simulation methods for ionic liquid electric double layers

The Journal of Chemical Physics 144, 184707 (2016); 10.1063/1.4948938

Revisiting density functionals for the primitive model of electric double layers

The Journal of Chemical Physics 140, 044714 (2014); 10.1063/1.4862990

\section{AIP | The Jounal of Chemical Physics}

PERSPECTIVES 


\title{
Time-dependent density functional theory for the charging kinetics of electric double layer containing room-temperature ionic liquids
}

\author{
Cheng Lian, ${ }^{1,2}$ Shuangliang Zhao, ${ }^{1}$ Honglai Liu, ${ }^{1, a)}$ and Jianzhong $\mathrm{Wu}^{2, b)}$ \\ ${ }^{1}$ State Key Laboratory of Chemical Engineering, and School of Chemistry and Molecular Engineering, \\ East China University of Science and Technology, Shanghai, 200237, People's Republic of China \\ ${ }^{2}$ Department of Chemical and Environmental Engineering, University of California, Riverside, \\ California 92521, USA
}

(Received 19 August 2016; accepted 7 November 2016; published online 29 November 2016)

\begin{abstract}
Understanding the charging kinetics of electric double layers is of fundamental importance for the design and development of novel electrochemical devices such as supercapacitors and field-effect transistors. In this work, we study the dynamic behavior of room-temperature ionic liquids using a classical time-dependent density functional theory that accounts for the molecular excluded volume effects, the electrostatic correlations, and the dispersion forces. While the conventional models predict a monotonic increase of the surface charge with time upon application of an electrode voltage, our results show that dispersion between ions results in a non-monotonic increase of the surface charge with the duration of charging. Furthermore, we investigate the effects of van der Waals attraction between electrode/ionic-liquid interactions on the charging processes. Published by AIP Publishing. [http://dx.doi.org/10.1063/1.4968037]
\end{abstract}

\section{INTRODUCTION}

Room temperature ionic liquids (RTILs) have been widely used as environmentally friendly solvents for separation, catalysts, and electrochemical processes. Their use as electrolytes in electrochemical devices including batteries and supercapacitors has been studied extensively. ${ }^{1}$ In comparison to aqueous electrolytes, RTILs have major advantages owing to their large electrochemical windows, excellent thermal stability, and low volatility. ${ }^{2}$ The equilibrium structure of electric double layers (EDLs) in RTILs is relatively well understood by previous experimental and theoretical investigations. ${ }^{1,3-12}$ The EDL structure of ionic liquids exhibits alternating layers of cations and anions distributed near a charged surface with the peak densities decaying to the corresponding bulk values at a length scale up to 10 times the ionic diameters. The layering structure is responsible for the oscillatory variation of the capacitance with the pore size. ${ }^{13-18}$

Despite vast literatures on the equilibrium or static behavior of RTILs at charged interfaces, understanding the dynamics of RTILs in response to an applied electrode surface charge or potential is difficult from both experimental and theoretical perspectives. A number of experimental techniques have been used to study the dynamic properties; common methods include NMR spectroscopy, ${ }^{19,20}$ electrochemical quartz crystal microbalance (EQCM) ${ }^{21} \mathrm{XRD},{ }^{22}$ and infrared spectroelectrochemistry. ${ }^{23}$ From a theoretical perspective, molecular dynamics (MD) simulations are commonly used to study the charging dynamics of RTILs in porous materials. ${ }^{24-29}$ The simulation results indicate that the charging of a pore pre-wetted by RTILs is a diffusive process ${ }^{24}$ and that an ionophobic pore can accelerate the charging and cyclic recharging. ${ }^{25}$ Qualitatively,

\footnotetext{
a)E-mail: hlliu@ecust.edu.cn

b) E-mail: jwu@engr.ucr.edu
}

the simulation results can be recaptured by of mean-field models. ${ }^{26}$ Péan et al. studied the influence of the structure of the electrode materials and the effects of potential and solvation on the dynamic processes. ${ }^{27,28}$ The MD simulations of the cyclic voltammetry of subnanometer pores with RTILs show that the cyclic charging and discharging of nanopores are governed by the competition between the external field-driven ion transport and the sloshing dynamics of ions inside of the pore. $^{29}$

Analytical methods ${ }^{30-40}$ are computationally more efficient than MD simulations thereby suitable for a systematic investigation of the key parameters for relatively large systems. In particular, time-dependent density functional theory (TDDFT) can be used to account for the ionic steric effects and electrostatic correlations neglected in the Poisson-NernstPlanck (PNP) equations, a conventional microscopic theory for EDL charging. The non-mean-field effects are significant for RTILs due to strong electrostatic interactions and high ion densities. ${ }^{41-43}$ While steric interactions and electrostatic correlations have received much attention in the recent literatures, the van der Waals attractions are often not included in the coarse-grained models for RTILs. Because dispersion forces play an important role on the bulk properties of the ionic liquids, their effects may not be negligible at interfaces and lead to a different mechanism for EDL charging process. In this work, we extend the TDDFT proposed in our previous work ${ }^{36,42}$ to examine the effect of dispersion interaction on the charging behavior of EDLs containing RTILs.

\section{MODEL AND METHODS}

Our theoretical investigation of electric double layer (EDL) charging is based on the nonprimitive model for a roomtemperature ionic liquid (RTIL) confined between two parallel planar electrodes, as shown schematically in Figure 1. This 


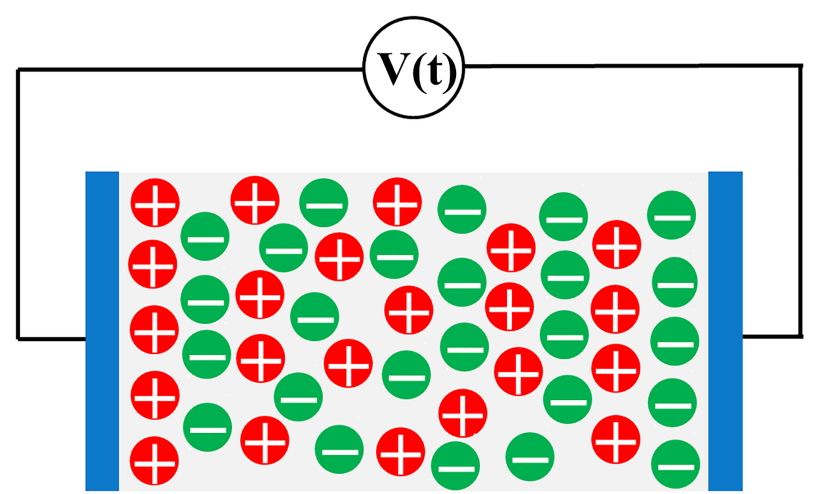

FIG. 1. A model electrochemical cell for studying the charging behavior of EDLs. The nonprimitive model is used to represent a room-temperature ionic liquid wherein both cations and anions are approximated by monovalent charged hard spheres.

model has been successfully used by Jiang et al. to study the effect of pore size on the EDL capacitance. ${ }^{44}$ Approximately, the electrolyte system mimics 1-ethyl-3-methylimidazolium bis-(trifluoromethanesulfonyl) imide (EMI-TFSI), a common RTIL for supercapacitor studies. The ion diameter and the valence are $\sigma=0.5 \mathrm{~nm}$ (corresponding to the averaged size of EMI and TFSI ${ }^{14}$ ) and $Z_{i}= \pm 1$, respectively. At $298 \mathrm{~K}$ and 1 bar, the molar volume and diffusion coefficient for the ionic liquid are $v=259 \mathrm{~cm}^{3} / \mathrm{mol}$ and $D=4.29 \times 10^{-11} \mathrm{~m}^{2} / \mathrm{s}$, respectively. ${ }^{14}$

Time-dependent density functional theory (TDDFT) is an extension of the classical density functional theory (DFT) to describe dynamic or time-dependent processes based on the assumption of local thermodynamic equilibrium. ${ }^{45-53}$ For ion diffusion in an electrolyte solution near electrodes, TDDFT asserts that the time evolution for the local density profiles of ionic species, $\rho_{i}(\mathbf{r}, t)$, follows the generalized diffusion equation

$$
\frac{\partial \rho_{i}(\mathbf{r}, t)}{\partial t}=\nabla \cdot\left\{D_{i} \rho_{i}(\mathbf{r}, t) \nabla\left[\beta \mu_{i}(\mathbf{r}, t)+\beta V_{i}(\mathbf{r})\right]\right\},
$$

where $D_{i}$ stands for the self-diffusivity of ion $i, \beta=1 /\left(k_{B} T\right)$, $k_{B}$ is the Boltzmann constant, $T$ stands for the absolute temperature, $\mu_{i}(\mathbf{r}, t)$ is the local chemical potential, and $V_{i}(\mathbf{r})$ denotes the external potential arising from the electrodes. In electrochemistry, the local chemical potential, together with the electrostatic component of the external potential, is often referred to as the electrochemical potential, here designated as $u_{i}(\mathbf{r}, t) \equiv \mu_{i}(\mathbf{r}, t)+V_{i}(\mathbf{r})$.

Conventionally, the electrochemical potential is replaced by that for an ideal solution plus an additional term due to the mean electric potential, $\psi(\mathbf{r}, t)$, i.e.,

$$
u_{i}(\mathbf{r}, t)=k_{B} T \ln \left[\rho_{i}(\mathbf{r}, t) \Lambda_{i}^{3}\right]+Z_{i} e \psi(\mathbf{r}, t),
$$

where $Z_{i}$ is the ion valence, $e$ represents the unit charge, and $\Lambda_{i}$ denotes an effective thermal wavelength of ion $i$. Within the ideal solution model, Eq. (1) reduces to the celebrated Nernst-Planck (NP) equation for ion diffusion in an electric field, ${ }^{54}$

$$
\frac{\partial \rho_{i}(\mathbf{r}, t)}{\partial t}=\nabla \cdot\left\{D_{i} \nabla \rho_{i}(\mathbf{r}, t)+\frac{Z_{i} e D_{i} \rho_{i}(\mathbf{r}, t)}{k_{B} T} \nabla \psi(\mathbf{r}, t)\right\} .
$$

Eq. (3) can be solved in conjunction with the Poisson equation for the electric potential

$$
\nabla^{2} \psi(\mathbf{r}, t)=-\frac{e}{\varepsilon_{r} \varepsilon_{0}} \sum_{i} Z_{i} \rho_{i}(\mathbf{r}, t),
$$

where $\varepsilon_{0}$ is the permittivity of the free space, and $\varepsilon_{r}$ is the dielectric constant. The NP equation ignores interaction between ionic species other than the direct Coulomb interaction. Similar to that used in the Poisson-Boltzmann equation for predicting the equilibrium properties of electrolyte solutions, the assumptions leading to the NP equation are valid only at low salt concentration. To account for non-electrostatic interactions and correlation effects, we need a more realistic expression for $\mu_{i}(\mathbf{r}, t)$.

According to the model of electrolyte solutions, ${ }^{55-58}$ the electrochemical potential for each ionic species includes, in addition to the ideal-solution term and the mean electrostatic potential $\psi(\mathbf{r}, t)$, contributions from the molecular excluded volume effects, the electrostatic correlations, and dispersion forces,

$$
\begin{aligned}
u_{i}(\mathbf{r}, t)= & k_{B} T \ln \left[\rho_{i}(\mathbf{r}, t) \Lambda_{i}^{3}\right]+e Z_{i} \psi(\mathbf{r}, t) \\
& +\mu_{\mathrm{ex}, i}^{\mathrm{HS}}(\mathbf{r}, t)+\mu_{\mathrm{ex}, i}^{\mathrm{DIS}}(\mathbf{r}, t)+\mu_{\mathrm{ex}, i}^{\mathrm{EC}}(\mathbf{r}, t) .
\end{aligned}
$$

In Eq. (5), $\mu_{\mathrm{ex}, i}^{\mathrm{HS}}(\mathbf{r}, t)$ is the local excess chemical potential due to hard sphere (HS) repulsion, $\mu_{\mathrm{ex}, i}^{\mathrm{DIS}}(\mathbf{r}, t)$ represents the contribution of dispersion forces (DIS), and $\mu_{\mathrm{ex}, i}^{\mathrm{EC}}(\mathbf{r}, t)$ represents an additional contribution due to the electrostatic correlations (EC).

As in our previous work for electrolyte systems at equilibrium, we use the modified fundamental measure theory (MFMT) for $\mu_{\mathrm{ex}, i}^{\mathrm{HS}}(\mathbf{r}, t),{ }^{59}$ the mean-field approximation for $\mu_{\mathrm{ex}, i}^{\mathrm{DIS}}(\mathbf{r}, t),{ }^{60}$ and a second-order perturbation theory for $\mu_{\mathrm{ex}, i}^{\mathrm{EC}}(\mathbf{r}, t) .{ }^{61}$ The numerical performance of the equilibrium methods has been carefully calibrated with molecular simulations. ${ }^{58,61}$

According to MFMT, the excess chemical potential due to hard-sphere interactions is given by

$$
\beta \mu_{\mathrm{ex}, i}^{\mathrm{HS}}(\mathbf{r})=\sum_{\alpha} \int \mathrm{d} \mathbf{r}^{\prime} \xi_{\alpha}\left(\mathbf{r}^{\prime}\right) \omega_{i}^{(\alpha)}\left(\mathbf{r}^{\prime}-\mathbf{r}\right),
$$

where

$$
\begin{gathered}
\xi_{0}=-\ln \left(1-n_{3}\right), \\
\xi_{1}=\frac{n_{2}}{1-n_{3}},
\end{gathered}
$$

$$
\begin{gathered}
\xi_{2}=\frac{n_{1}}{1-n_{3}}+\left[\frac{\ln \left(1-n_{3}\right)}{n_{3}}+\frac{1}{\left(1-n_{3}\right)^{2}}\right] \frac{n_{2}^{2}-\mathbf{n}_{V 2}^{2}}{12 \pi n_{3}} \\
\xi_{3}=-\left[\frac{\ln \left(1-n_{3}\right)}{18 \pi n_{3}^{3}}+\frac{1-3 n_{3}+\left(1-n_{3}\right)^{2}}{36 \pi n_{3}^{2}\left(1-n_{3}\right)^{3}}\right]\left(n_{2}^{3}-3 n_{2} \mathbf{n}_{V 2}^{2}\right) \\
+\frac{n_{0}}{1-n_{3}}+\frac{n_{1} n_{2}-\mathbf{n}_{V 1} \mathbf{n}_{V 2}}{\left(1-n_{3}\right)^{2}},
\end{gathered}
$$




$$
\begin{gathered}
\xi_{V 1}=-\frac{\mathbf{n}_{V 2}}{1-n_{3}}, \\
\xi_{V 2}=-\frac{\mathbf{n}_{V 1}}{1-n_{3}}-\left[\frac{\ln \left(1-n_{3}\right)}{n_{3}}+\frac{1}{\left(1-n_{3}\right)^{3}}\right] \frac{n_{2} \mathbf{n}_{V 2}}{6 \pi n_{3}} .
\end{gathered}
$$

The detail expressions for the weighted densities $\left\{n_{\alpha}\right\}$ and the corresponding weighting functions $\left\{\omega_{\alpha}\right\}$ are given in the previous publications. $57-59,61-63$

The dispersion interaction between species $i$ and $j$ is described by the square-well (SW) potential $u_{i j}^{\mathrm{DIS}}(r)=-\varepsilon_{i j}$ for $r<\lambda_{i j}$ with $\lambda_{i j}$ being the range of attraction. Although the square-well potential is different to the London dispersion interaction, they both capture the feature of short-range attraction between two ions. The excess chemical potential due to the dispersion attraction is approximated by the mean-field approximation

$$
\beta \mu_{\mathrm{ex}, i}^{\mathrm{DIS}}(\mathbf{r})=\frac{1}{2} \sum_{j=+,-} \int \rho_{j}\left(\mathbf{r}^{\prime}\right) u_{i j}^{\mathrm{DIS}}\left(\left|\mathbf{r}-\mathbf{r}^{\prime}\right|\right) \mathrm{d} \mathbf{r}^{\prime} .
$$

The excess chemical potential due to electrostatic correlation is given by

$\beta \mu_{\mathrm{ex}, i}^{\mathrm{EC}}(\mathbf{r})=\beta \mu_{\mathrm{b}, i}^{\mathrm{EC}}-\sum_{j=+,-} \int \Delta \rho_{j}\left(\mathbf{r}^{\prime}\right) c_{i j}^{\mathrm{EC}}\left(\left|\mathbf{r}-\mathbf{r}^{\prime}\right|\right) \mathrm{d} \mathbf{r}^{\prime}$,

where $\mu_{\mathrm{b}, i}^{\mathrm{EC}}$ represents the excess chemical potential due to electrostatic correlation of specie $i$ in a bulk reference system, and $c_{i j}^{E C}(r)$ is direct correlation function of the bulk fluid due to electrostatic correlations. We calculate $c_{i j}^{E C}(r)$ from the meanspherical approximation, ${ }^{64}$ details are given in the previous publications. ${ }^{57-59,61,62}$

Given the initial density profiles of the ionic species calculated from the equilibrium DFT, Eq. (1) can be integrated numerically along with the Poisson equation. With fixed voltages at the cell boundaries, the spatial variation of the electrostatic potential can be calculated from the instantaneous ionic density profiles.

The mathematical details for numerical integration of the TDDFT equation can be found in our previous publication. ${ }^{42}$ The ionic density profiles can be discretized in terms of differential time frames. Within the duration of a time step starting from $t_{k}$ to $t_{k+1}=t_{k}+\Delta t$, the equation of continuity for each ionic species may be casted as an ordinary differential equation

$$
\frac{\partial \rho_{i}(\mathbf{r}, t)}{\partial t}=\mathbf{M}_{i}\left[\left\{\rho_{i}\left(\mathbf{r}, t_{k}\right)\right\}, t\right] .
$$

The right side of Eq. (15) is related to the ionic flux and subsequently the electrochemical potential

$$
\begin{aligned}
\mathbf{M}_{i}\left[\left\{\rho_{i}\left(\mathbf{r}, t_{k}\right)\right\}, t\right] & =-\nabla \cdot \mathbf{j}_{i}\left(\mathbf{r}, t_{k}\right) \\
& =\nabla \cdot\left\{\lambda_{i} \rho_{i}\left(\mathbf{r}, t_{k}\right) \nabla\left[\varphi_{i}\left(\mathbf{r}, t_{k}\right)+V_{i}^{0}\left(\mathbf{r}, t_{k}\right)\right]\right\} .
\end{aligned}
$$

Given an expression for $\varphi_{i}\left(\mathbf{r}, t_{k}\right)$ the electrochemical potential, we can readily integrate Eq. (15) with the fourth-order
Adams-Moulton (AM) algorithm

$$
\begin{aligned}
\rho_{i}\left(\mathbf{r}, t_{k+1}\right)= & \rho_{i}\left(\mathbf{r}, t_{k}\right)+\frac{\Delta t}{24}\left(9 \mathrm{M}_{i}\left[\left\{\rho_{i}\left(\mathbf{r}, t_{k+1}\right)\right\}, t_{k+1}\right]\right. \\
& +19 \mathrm{M}_{i}\left[\left\{\rho_{i}\left(\mathbf{r}, t_{k}\right)\right\}, t_{k}\right] \\
& -5 \mathrm{M}_{i}\left[\left\{\rho_{i}\left(\mathbf{r}, t_{k-1}\right)\right\}, t_{k-1}\right] \\
& \left.+\mathrm{M}_{i}\left[\left\{\rho_{i}\left(\mathbf{r}, t_{k-2}\right)\right\}, t_{k-2}\right]\right) .
\end{aligned}
$$

In numerical evaluation of function $\mathrm{M}_{i}$ from Eq. (16), we calculate both the divergence and the gradient using the finite difference method. Meanwhile, the electrostatic potential is calculated by the numerical integration of the Poisson equation with the boundary conditions specified by the electric potentials at the electrodes.

We find that the AM algorithm yields excellent numerical stability and accuracy except that the implicit method requires the numerical solution of a set of nonlinear equations at every time step. To accelerate solution of $\rho_{i}\left(\mathbf{r}, t_{k+1}\right)$ with the Picard iteration, we use an initial guess from the fourth-order AdamsBashforth $(\mathrm{AB})$ predictor,

$$
\begin{aligned}
\rho_{i}^{(0)}\left(\mathbf{r}, t_{k+1}\right)= & \rho_{i}\left(\mathbf{r}, t_{k}\right)+\frac{\Delta t}{24}\left(55 \mathrm{M}_{i}\left[\left\{\rho_{\alpha}\left(\mathbf{r}, t_{k}\right)\right\}, t_{k}\right]\right. \\
& -59 \mathrm{M}_{i}\left[\left\{\rho_{i}\left(\mathbf{r}, t_{k-1}\right)\right\}, t_{k-1}\right] \\
& +37 \mathrm{M}_{i}\left[\left\{\rho_{i}\left(\mathbf{r}, t_{k-2}\right)\right\}, t_{k-2}\right] \\
& \left.-9 \mathrm{M}_{i}\left[\left\{\rho_{i}\left(\mathbf{r}, t_{k-3}\right)\right\}, t_{k-3}\right]\right) .
\end{aligned}
$$

With an initial guess provided by the AB method, the Picard iteration typically converges within only few loops.

\section{RESULTS AND DISCUSSION}

In our TDDFT calculations, the reduced density of bulk ionic liquid is set as $\rho_{\mathrm{b}}^{*}=\rho_{+} \sigma^{3}=\rho_{-} \sigma^{3}=0.29$, which is consistent with the molar volume for the model ionic liquid $\left(v=259 \mathrm{~cm}^{3} / \mathrm{mol}\right)$. The separation between two electrodes is fixed at $10 \mathrm{~nm}$, which is large enough to eliminate the pore size effect. ${ }^{44,65}$ The electrodes are kept neutral at $t<0$ and applied with a constant voltage $\pm 0.3 \mathrm{~V}$ during the charging process $(t \geq 0)$. Throughout this work, the time is given in units of $\tau_{\mathrm{D}}=\sigma^{2} / D$, where $D$ represents ion self-diffusivity. For the EMI-TFSI model, $\tau_{\mathrm{D}}$ is $\sim 5.83 \mathrm{~ns}$.

Figures 2(a) and 2(b) show how the cation and anion density profiles change with the charging time without accounting for dispersion forces. After applying a constant voltage, the contact density of cations increases, concomitant with the reduction of the contact density for anions. As increasing the duration of charging, TDDFT predicts formation of alternating layers of cations and anions near the charged electrode. The layer-by-layer structure arises from charge inversion due to strong electrostatic correlations and the enhanced excluded-volume effect. ${ }^{44}$

For comparison, the evolutions of ionic density profiles that account for the dispersion among ionic species are shown in Figures 2(c) and 2(d). Here the dispersion energy is calculated using $\varepsilon=0.7 k_{\mathrm{B}} T$. By comparing the 

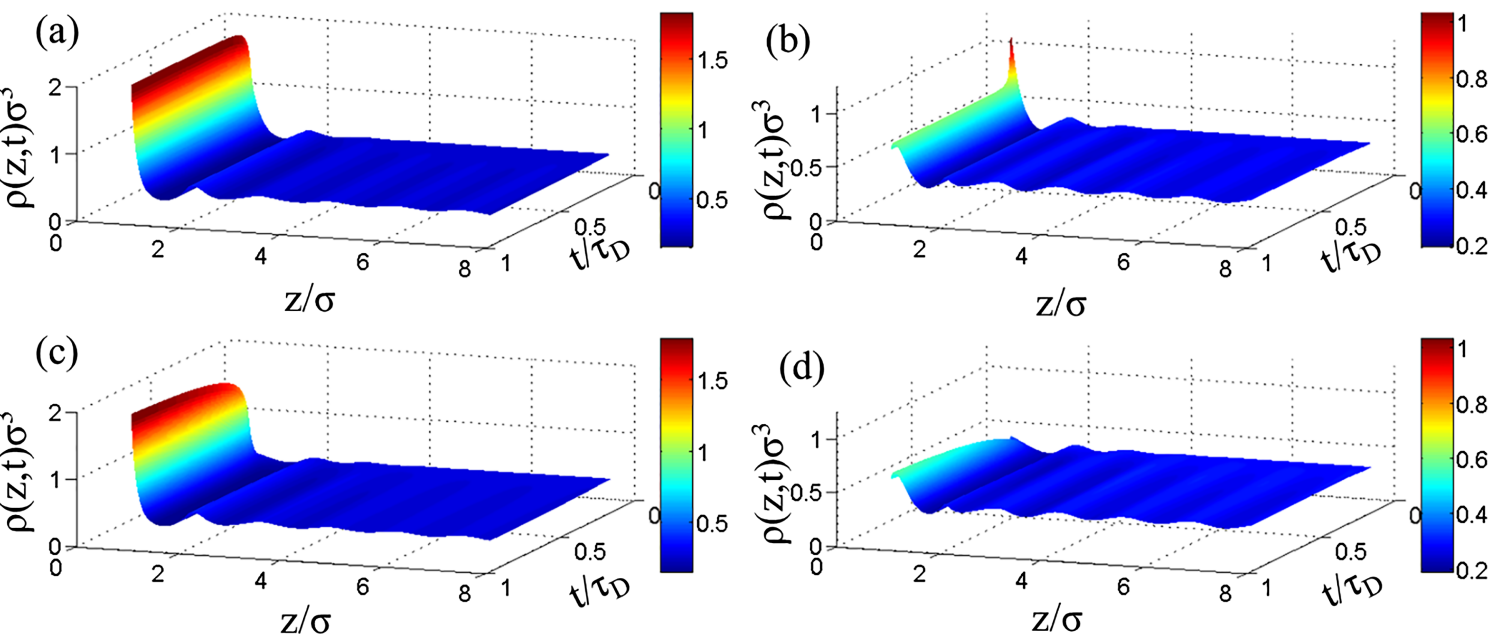

FIG. 2. Evolution of the local number density for cations (a) and (c) and anions (b) and (d) near the electrode surface during constant-voltage charging. No dispersion interaction for (a) and (b); with dispersion interaction $\varepsilon=0.7 k_{\mathrm{B}} T$ for (c) and (d).

density evolutions in different panels, one can find from Figure 2 that the dispersion force only changes the ions near the electrode surface at the beginning stages of the EDL charging.

To examine the dispersion effect on the EDL structure, we present in Figure 3 the ionic density profiles at $t=0$ and $\infty$. Regardless of the surface charge, the density profile near the electrode always exhibits a certain degree of inhomogeneity. Such inhomogeneity arises from ionic excluded volume effects and electrostatic correlations which is not captured by the conventional Poisson-Boltzmann equation (PBE). The contact density for $\varepsilon=0.7 k_{\mathrm{B}} T$ is smaller than that for $\varepsilon=0.0$ due to the dispersion attraction from the bulk. As charging proceeds, formation of a counterion layer near the electrode over compensates the surface charge, leading to charge inversion and oscillatory ionic distributions, as shown in Figure 3(b). Interestingly, the dispersion energy has little effect on the equilibrium density profiles near a charged surface. Here the relatively minor effect of dispersion is expected because the Coulombic interaction between ions is far larger than the dispersion interaction in this layer-by-layer equilibrium structure, and the Coulombic interaction dominates the ion-surface interactions.

A key measure of practical interest is the surface charge density, which is related to the ionic density distributions

$$
Q(t)=-\int_{0}^{L / 2} \sum_{i} Z_{i} e \rho_{i}(z, t) \mathrm{d} z,
$$

where $L$ denotes the separation between two parallel electrodes. Conventionally, the EDL charging is described with the equivalent circuit (EC) model, which predicts a monotonic increase of the surface charge density according to ${ }^{66}$

$$
Q(t)=Q_{0}\left(1-e^{-t / \tau}\right),
$$

where $Q_{0}$ stands for the charge density at the electrode surface at equilibrium, $\tau$ represents the response time reflecting the rate of charging process.

Figure 4 shows that the surface charge density changes with the charging time at different strengths of dispersion interactions. Without considering the dispersion effect, the surface charge increases monotonically until the equilibrium value, which is in reasonable agreement with the prediction of the EC model. As the dispersion force increases, one can see that the surface charge rises quickly with time to a maximum and then decays monotonically to the equilibrium value. At the beginning stage of charging, the counterions form a contact layer to balance the surface potential, coions need to overcome the dispersion force to arrive from the bulk to screen the counterion contact layer, and the surface charge with dispersion force is larger than that without dispersion. As charging proceeds, the electric interaction dominates the process, the surface charge

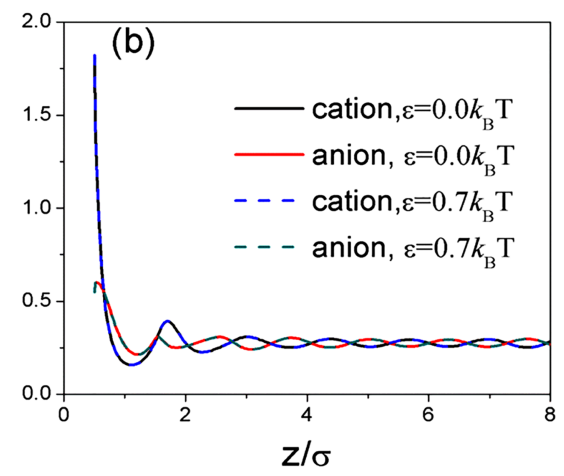

FIG. 3. Reduced density profiles of cations or anions when $t=0$ (a) and $t=\infty$ (b). 


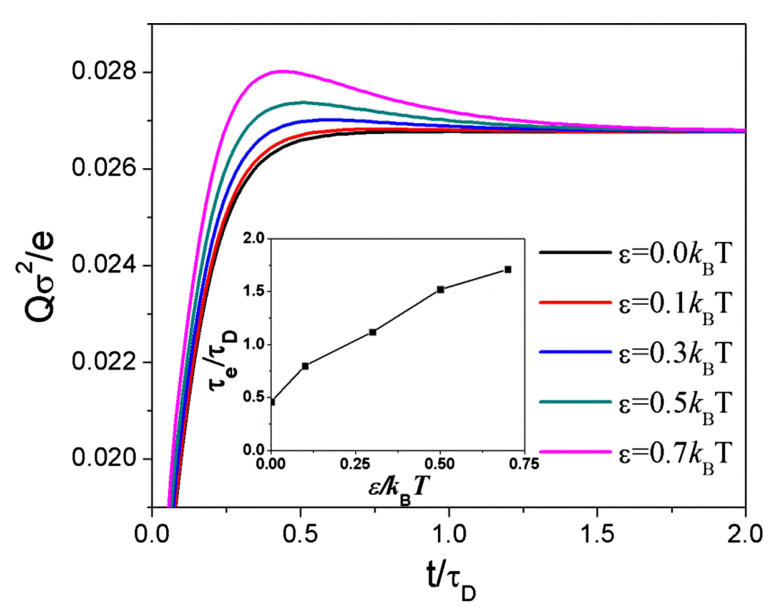

FIG. 4. Evolution of surface charge density for different strengths of dispersion forces. (Inset) The time towards equilibrium $\tau_{\mathrm{e}} / \tau_{\mathrm{D}}$ as a function of the dispersion energy parameter $\varepsilon$.

decreases to equilibrium value. The time towards equilibrium $\tau_{\mathrm{e}} / \tau_{\mathrm{D}}$ is illustrated in the inset of Figure 4 . The dispersion interaction controls the extent of overcompensation of counterions contact layer charge by the arriving of coions from the bulk. Therefore, increasing the dispersion force reduces the extent of charge overcompensation and increases the surface charge and the time towards equilibrium.

Next, we investigate the role of dispersion energy between the ionic-liquid and the electrodes. For simplicity, we use the square-well $(\mathrm{SW})$ potentials to describe the dispersive (nonelectrostatic) surface potential,

$V^{D I S}(z)=\left\{\begin{array}{cc}\infty, & z \leq \frac{\sigma_{i}}{2} \text { or } z \geq H-\frac{\sigma_{i}}{2} \\ -\varepsilon_{w}, & \frac{\sigma_{i}}{2}<z<\sigma_{i} \text { or } H-\sigma_{i}<z<H-\frac{\sigma_{i}}{2}, \\ 0, & \text { otherwise }\end{array}\right.$

where the dispersion force (attraction) is regulated by the surface energy $\varepsilon_{w} \cdot \varepsilon_{w}>0$ represents an attractive potential, and $\varepsilon_{w}=0$ represents a hard wall.

Figure 5 shows the ionic density profiles at $t=0$ with two different electrodes. As expected, the surface attraction

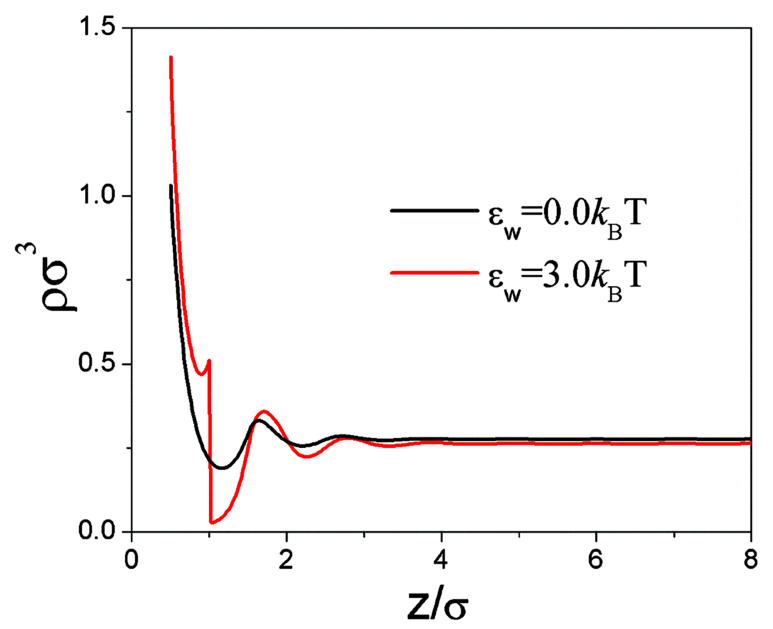

FIG. 5. The ionic density profiles at $t=0$ with different surface energies. The black line is for the system without ion-electrode dispersion energy $\left(\varepsilon_{\mathrm{W}}=0\right)$, and the red line is for the system with a surface energy, $\varepsilon_{\mathrm{W}}=3.0 k_{\mathrm{B}} T$.

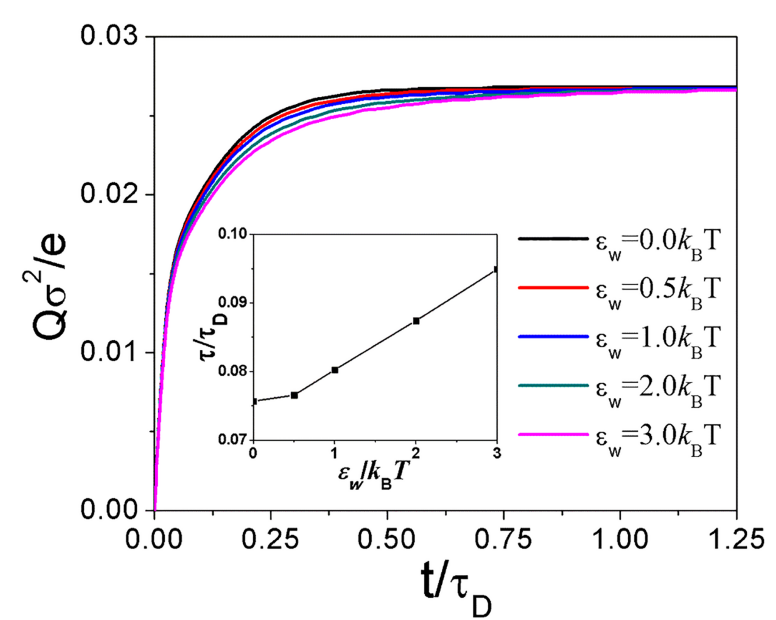

FIG. 6. The surface charge density as a function of time during EDL charging for various wall-ion dispersion energy $\varepsilon_{\mathrm{W}}$. (Inset) The relaxation time towards equilibrium $\tau_{\mathrm{e}} / \tau_{\mathrm{D}}$ as a function of the wall-ion dispersion energy $\varepsilon_{\mathrm{W}}$.

makes more ions adsorption near the electrodes. The effect of surface attraction on the charging process is shown in Figure 6. Unlike the ion-ion dispersion effect, the electrode dispersion has little influence on the charging behavior. As for the system with or without surface dispersion, TDDFT predicts a monotonic evolution of the surface charge density. The wall-ion dispersion force also has no effects on the equilibrium properties, due to the Coulombic interaction is far larger than the dispersion interaction at charged surface, and the Coulombic interaction dominates the ion-surface interactions. The relaxation time towards equilibrium $\tau / \tau_{\mathrm{D}}$ as a function of the wall-ion dispersion interaction $\varepsilon_{\mathrm{W}}$ is shown in the inset of Figure 6. Here the relaxation time $\tau / \tau_{\mathrm{D}}$ is obtained by fitting the numerical data to EC model. The dispersion force between the electrode and ions increases the relaxation time because the dispersion force prevents the rearrangement of ions and increases the time of charging process. Our results are qualitatively agreed with the results of a meanfield model, which suggests that the charging of a pore prewetted by RTILs is a diffusive process ${ }^{24}$ and ionophobic pore can accelerate charging. ${ }^{25}$ In additional, we also consider different

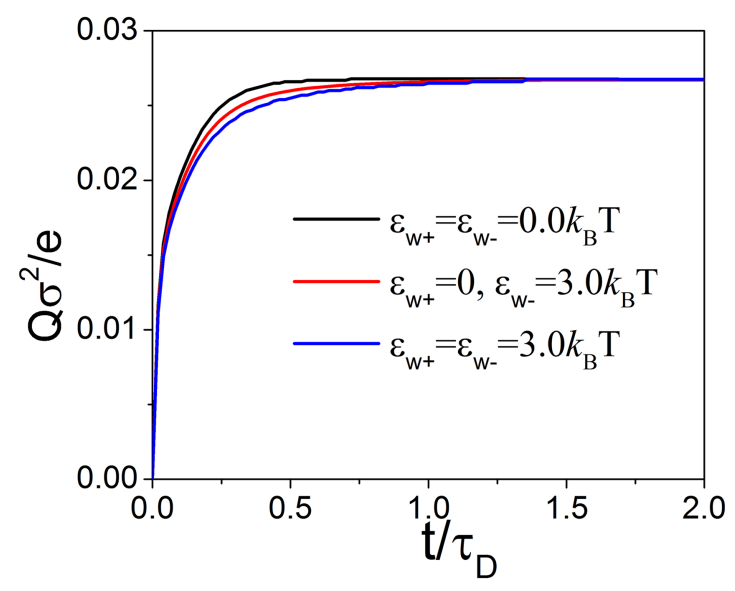

FIG. 7. The surface charge density as a function of time during EDL charging for various wall-ion dispersion energy $\varepsilon_{\mathrm{W}}$. Here, the wall-ion dispersion energy for cations $\left(\varepsilon_{\mathrm{W}_{+}}\right)$and anions $\left(\varepsilon_{\mathrm{W}_{-}}\right)$are different. 
ion-wall dispersion interactions for cations and anions, which is more reasonable than with the same dispersion interaction. The effect of surface attractions for cations and anions on the charging process is shown in Figure 7. As the result in Figure 6 with the same surface attraction, the dispersion between anion-wall also delays the charging at the beginning of charging process. Like Figure 6, the different wall-ion dispersion force for cations and anions also has no effects on the equilibrium properties, which means the ion-wall dispersion force has no effects on the equilibrium capacitance.

\section{CONCLUSIONS}

We have studied the charging behavior of electric double layers containing a model room-temperature ionic liquid (RTIL) using the classical time-dependent density functional theory (TDDFT). Different from the conventional PoissonNernst-Planck (PNP) equation, our TDDFT is able to account for the molecular excluded volume effects, electrostatic correlations, and the dispersion interactions. While the conventional models of EDL charging predict a monotonic increase of the surface charge with time upon application of an electrode voltage, our results show that the dispersion interaction between ions makes the surface charge be a non-monotonic function of time. However, the dispersion interaction between the electrode and ionic-liquid does not change the monotonic evolution of surface charge density. Both the ion-ion and the electrodeion dispersion interactions increase the duration of the EDL charging process. We hope this theoretical work enables a way to tune the charging behavior of electric double layer capacitors (EDLCs) by an appropriate choice ofelectrodes and ionic liquids.

\section{ACKNOWLEDGMENTS}

This research was sponsored by the Fluid Interface Reactions, Structures and Transport (FIRST) Center, an Energy Frontier Research Center funded by the U.S. Department of Energy, Office of Science, Office of Basic Energy Sciences. Cheng Lian and Honglai Liu thank the financial support by the National Natural Science Foundation of China (Grant No. 91334203) and the 111 Project of China (Grant No. B08021). Cheng Lian is also grateful to the Chinese Scholarship Council for the visiting fellowship. The numerical calculations were performed at the National Energy Research Scientific Computing Center (NERSC).

${ }^{1}$ R. Hayes, G. G. Warr, and R. Atkin, Chem. Rev. 115, 6357 (2015).

${ }^{2}$ K. R. Seddon, J. Chem. Technol. Biotechnol. 68, 351 (1997).

${ }^{3}$ P. Simon and Y. Gogotsi, Acc. Chem. Res. 46, 1094 (2012).

${ }^{4}$ R. Burt, G. Birkett, and X. Zhao, Phys. Chem. Chem. Phys. 16, 6519 (2014).

${ }^{5}$ C. Zhong et al., Chem. Soc. Rev. 44, 7484 (2015).

${ }^{6}$ D.-e. Jiang and J. Wu, J. Phys. Chem. Lett. 4, 1260 (2013).

${ }^{7}$ Y. Shao et al., Chem. Soc. Rev. 44, 3639 (2015).

${ }^{8}$ M. V. Fedorov and A. A. Kornyshev, Chem. Rev. 114, 2978 (2014).
${ }^{9}$ E. Frackowiak, Q. Abbas, and F. Béguin, J. Energy. Chem. 22, 226 (2013).

${ }^{10} \mathrm{G}$. Feng et al., AlChE J. 61, 3022 (2015).

${ }^{11}$ K. M. Gupta et al., ACS Appl. Mater. Interfaces 8, 13392 (2016).

${ }^{12} \mathrm{G}$. Feng et al., "Structure of the electrical double layers: Insights from Continuum and Atomistic Simulations," in Computational Bioengineering, edited by G. Zhang (CRC Press, 2015), pp. 327-354.

${ }^{13}$ M. Mezger et al., Science 322, 424 (2008).

${ }^{14}$ C. Largeot et al., J. Am. Chem. Soc. 130, 2730 (2008).

${ }^{15}$ C. Lian et al., J. Phys. Chem. C 120, 8704 (2016).

${ }^{16} \mathrm{P}$. Wu et al., ACS Nano 5, 9044 (2011).

${ }^{17}$ C. Lian et al., ACS Energy Lett. 1, 21 (2016).

${ }^{18}$ G. Feng and P. T. Cummings, J. Phys. Chem. Lett. 2, 2859 (2011).

${ }^{19}$ J. M. Griffin et al., Nat. Mater. 14, 812 (2015).

${ }^{20}$ A. C. Forse et al., J. Am. Chem. Soc. 138, 5731 (2016).

${ }^{21}$ W.-Y. Tsai, P.-L. Taberna, and P. Simon, J. Am. Chem. Soc. 136, 8722 (2014).

${ }^{22}$ C. Prehal et al., Energy Environ. Sci. 8, 1725 (2015).

${ }^{23}$ F. W. Richey et al., J. Phys. Chem. C 118, 21846 (2014).

${ }^{24}$ S. Kondrat and A. Kornyshev, J. Phys. Chem. C 117, 12399 (2013).

${ }^{25}$ S. Kondrat, G. Oshanin, and A. A. Kornyshev, Nanotechnology 25, 315401 (2014).

${ }^{26}$ S. Kondrat et al., Nat. Mater. 13, 387 (2014).

${ }^{27}$ C. Péan et al., ACS Nano 8, 1576 (2014).

${ }^{28}$ C. Pean et al., Electrochim. Acta 206, 504 (2016).

${ }^{29}$ Y. He et al., J. Phys. Chem. Lett. 6, 22 (2015).

${ }^{30}$ A. A. Lee et al., Phys. Rev. Lett. 115, 106101 (2015).

${ }^{31}$ N. Gavish and A. Yochelis, J. Phys. Chem. Lett. 7, 1121 (2016).

${ }^{32}$ M. S. Kilic, M. Z. Bazant, and A. Ajdari, Phys. Rev. E 75, 021502 (2007).

${ }^{33}$ M. Z. Bazant et al., Adv. Colloid Interface Sci. 152, 48 (2009).

${ }^{34}$ A. Yochelis, Phys. Chem. Chem. Phys. 16, 2836 (2014).

${ }^{35}$ A. Yochelis, J. Phys. Chem. C 118, 5716 (2014).

${ }^{36}$ J. Jiang et al., J. Phys. Chem. Lett. 5, 2195 (2014).

${ }^{37}$ Y. Qiao, B. Tu, and B. Lu, J. Chem. Phys. 140, 174102 (2014).

${ }^{38}$ B. Lu et al., Commun. Comput. Phys. 3, 973 (2008).

${ }^{39}$ J. Song et al., Adv. Funct. Mater. 24, 1243 (2014).

${ }^{40}$ M. Ma, S. Zhao, and Z. Xu, Commun. Comput. Phys. 20, 441 (2016).

${ }^{41}$ P. H. Alijó et al., Electrochim. Acta 152, 84 (2015).

${ }^{42}$ J. Jiang et al., J. Phys.: Condens. Matter 26, 284102 (2014).

${ }^{43}$ P. H. Alijó et al., Fluid Phase Equilib. 362, 177 (2014).

${ }^{44}$ D.-e. Jiang, Z. Jin, and J. Wu, Nano Lett. 11, 5373 (2011).

${ }^{45}$ A. J. Archer, J. Chem. Phys. 130, 014509 (2009).

${ }^{46}$ S. L. Zhao and J. Z. Wu, J. Chem. Phys. 134, 054514 (2011).

${ }^{47}$ C. Lian et al., Langmuir 30, 4040 (2014).

${ }^{48}$ D. Stopper, R. Roth, and H. Hansen-Goos, J. Chem. Phys. 143, 181105 (2015).

${ }^{49}$ A. J. Archer and R. Evans, J. Chem. Phys. 121(9), 4246 (2004).

${ }^{50}$ U. M. B. Marconi and S. Melchionna, J. Chem. Phys. 131, 014105 (2009).

${ }^{51}$ A. J. Archer and M. Rauscher, J. Phys. A: Math. Gen. 37, 9325 (2004).

${ }^{52}$ J. Dzubiella and C. N. Likos, J. Phys.: Condens. Matter 15, L147 (2003).

${ }^{53}$ L. Cheng et al., J. Phys.: Condens. Matter 28, 414005 (2016).

${ }^{54}$ J. S. Newman and K. E. Thomas-Alyea, Electrochemical Systems, 3rd ed. (J. Wiley, Hoboken, N.J., 2004).

${ }^{55}$ D. Boda et al., J. Chem. Phys. 116, 7170 (2002).

${ }^{56}$ D. Gillespie, N. Valisko, and D. Boda, J. Phys.: Condens. Matter 17, 6609 (2005).

${ }^{57}$ Y. X. Yu, J. Z. Wu, and G. H. Gao, J. Chem. Phys. 120, 7223 (2004).

${ }^{58}$ Z. D. Li and J. Z. Wu, J. Phys. Chem. B 110, 7473 (2006).

${ }^{59}$ Y. X. Yu and J. Z. Wu, J. Chem. Phys. 117, 10156 (2002).

${ }^{60} \mathrm{~S}$. Zhao et al., Mesoscale Modeling Chem. Eng. 47, 1 (2016).

${ }^{61}$ Y. X. Yu, J. Z. Wu, and G. H. Gao, Chin. J. Chem. Eng. 12, 688 (2004).

${ }^{62}$ W. A. Fouad et al., Fluid Phase Equilib. 416, 62 (2016).

${ }^{63}$ L. Cheng et al., J. Phys.: Condens. Matter 28, 464008 (2016).

${ }^{64}$ L. Blum, Mol. Phys. 30, 1529 (1975).

${ }^{65}$ X. Kong et al., Nano Res. 8, 931 (2015).

${ }^{66}$ J. Newman and K. E. Thomas-Alyea, Electrochemical Systems (John Wiley \& Sons, 2012). 